\title{
Automatic Vehicle Identification for Multiple Purposes at Toll Collection System
}

\author{
Vinaya Singh P, P. A. Vijaya, Ravikumar
}

\begin{abstract}
With the major growth in roadways, there is a raise in the number of toll booths. These toll booths have lengthy queues, the time consumed in paying cash and returning change causes additional delay. In this paper, a system uses Radio Frequency Identification (RFID) technology, ARM LPC2148, GSM, relay and computer host. RFID is used to obtain the vehicle number. RFID card with unique id is mounted on every vehicle, data contain on the card is examined by the RFID scanner placed at the toll gate. If the vehicle belongs to authorized person or registered, fixed money is automatically deducted from the owners account, message will be send to registered mobile number and automatically toll gate is opened. In this project Rs.50 is deducted for car and Rs.100 is deducted for bus. If the account balance becomes insufficient then buzzer is alarmed, message will be sent to the owner that she/he has insufficient balance and should use manual toll payment. If the vehicle belongs to higher officials such as VIP, police, army, ministers or ambulance the toll gate is opened automatically and the amount is not deducted. For upcoming situation all vehicle information that passes the toll will be stored. Stolen vehicle directory is also present, if the card number of the vehicle matches then buzzer is alarmed, message will be sent to the registered police station, amount is deducted from owner's account and gate is opened. The advantage is that message is sent both to the owner and

police station. Coding is done in Embedded C. This system eliminate the manual cash handling, reduces traffic congestion and help in lesser fuel utilization. This makes automatic toll collection more convenient for the public use.
\end{abstract}

Keywords: Automatic vehicle identification, Embedded C, RFID, GSM, authorized user, VIP vehicle, stolen vehicle.

\section{INTRODUCTION}

If we offer good alternative for public transport which saves time, we won't have traffic problems in any country's economy. Presently highway plays an important function in economical development, with a development of economy the growth of highway is becoming faster. Traditional toll collection system is time consuming so there is a lengthy queue of vehicle to pass the toll plaza [1]. This is a technology for collection of toll to guarantee smooth flow of traffic in efficient and faster way. Automatic vehicle identification is used for multiple purposes.

Revised Version Manuscript Received on Jun 20, 2019.

Vinaya Singh P, M.Tech Student, Department of ECE, BNMIT, Bangaluru.

P. A. Vijaya, Professor and Head, Department of ECE, BNMIT, Bangaluru.

Ravikumar, Head Trainer, 5square, Bangaluru.
The system uses Radio Frequency Identification (RFID) equipment where RFID card can be either active or passive. Active card have their personal internal power supply permit a large range, while passive tag use incoming signal from scanner thus have a shorter range. Scanner will have an antenna to transmit also receive signal from each card. Here passive tags are used. RFID tag identifies the type of vehicle such as authorized vehicle or higher official's vehicle or stolen vehicle. Based on the type of vehicle some operations will be carried out. If the vehicle is authorized and registered then money is deducted from owner's account, message will be send to his mobile number regarding amount deducted as well as his balance and the toll gate is opened. If vehicle belongs to higher officials such as VIP, police, army, ministers or ambulance then amount is not deducted and toll gate is opened automatically. If vehicle is identified as stolen from the database maintained then the message is sent to the police station, a predetermined amount will be deducted and the gate is opened. Figure 1 shows the working module of automatic vehicle identification for multiple purposes at toll collection system. There are many uses of automatic vehicle identification for multiple purposes at toll collection system namely; reduction in time, reduction in cost, faster services. By keeping a balance on the card itself payments can be done, increase lane capacity, reduction in fuel consumption, reduces air pollution and convenience to users.

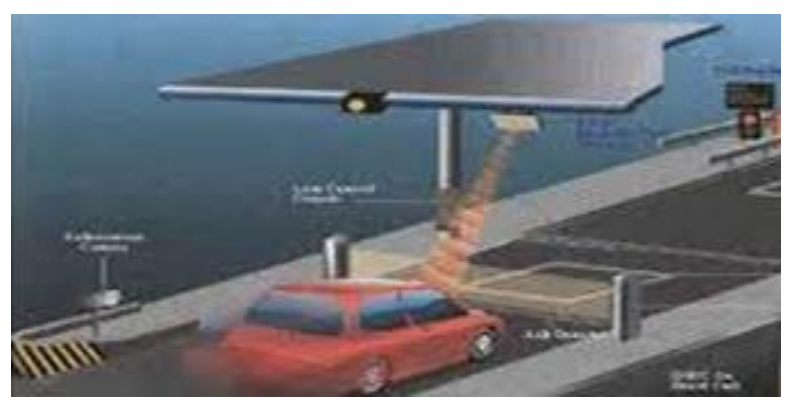

Figure 1: Working module

\section{BACKGROUND OVERVIEW}

\section{A. Existing System}

All motor vehicle have to stop at the toll booth next to highway, to give toll amount, one individual will gather cash and issue receipt. After receiving receipt gate is opened for driver to come out of toll plaza. This receipt is again checked by another person at the end of the toll for verification [2]. 


\section{B. Drawback of Existing System}

This system is slower and creates traffic jam as every vehicle has to stop; there are lengthy queue of motor vehicle at toll plaza. One person should be present to collect the toll amount and another for verification [3].

\section{Proposed System}

Automatic Vehicle Identification is a digital system for collecting toll from vehicles without stopping them. Toll collection lanes increase the speed and efficiency of traffic flow and saves drivers time. A RFID card is mounted on each vehicle with unique ID which contains all details about the vehicle and holder. When vehicle comes close to toll plaza, card will emit the radio wave signal. RFID scanner receives the signal from card, decodes that signal and sends to ARM controller. Microcontroller is interfaced with computer host to gather vehicle data through serial port for future use. Microcontroller checks balance if it has enough balance then fixed amount is deducted from the account. Using GSM modem message will be send to owners' mobile number regarding amount deducted and balance. The toll gate is opened. If the vehicle belongs to the higher officials then the toll gate is opened automatically. Here using RFID tag number we can determine if the vehicle belongs to higher officials or authorized user or stolen vehicle. If the vehicle is stolen then buzzer will be alarmed and message will be send to the registered police station. A predetermined amount is deducted from the owners account and then gate is opened. Figure 2 shows the proposed system. The key advantages over current system are automatic collection of toll tax, free flow of traffic, time saving and record preservation.

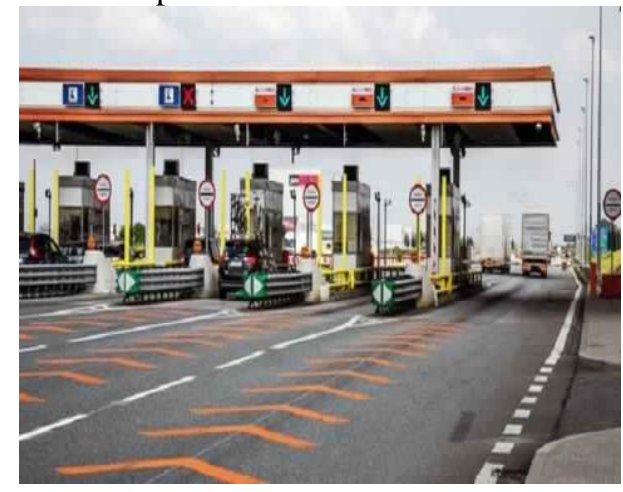

\section{Figure 2: Proposed System which reduces traffic}

\section{Block Diagram}

Block diagram of automatic vehicle identification for multiple purposes at toll collection system is as shown in Figure 3. RFID scanner decodes the information and send to controller. PC acts as a database and LCD displays the position of operation. GSM unit is interfaced through ARM controller which sends notification to car holder if he has inadequate amount or fewer amounts. Buzzer is used when vehicle is stolen. Motor driver is used for opening and closing the gate.

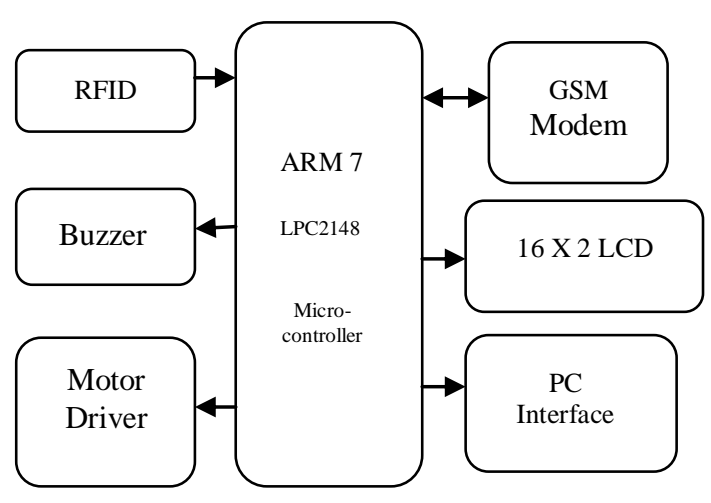

Figure 3: Block Diagram of Proposed

RFID (Kadio trequency identitication technology) is used for automatic detection and tracking of vehicle at toll booth which contains card and scanner. Data present on card is scanned by the reader and sent to the controller [5].

\section{E. RFID Reader}

RFID READER NSK EDK-125-TTL is used in this project and is shown in Figure 4. NSK125 series RFID reader module will have a built-in transmitter. It works with a typical carrier frequency of $125 \mathrm{kHz}$. To the host system the tag data is sent through the wired message interface using a protocol which is chosen from the component both TTL and Wiegand protocol. In this project TTL protocol is used.The LF component is best suited for applications in access control, occasion and attendance and extra RFID enabled applications.

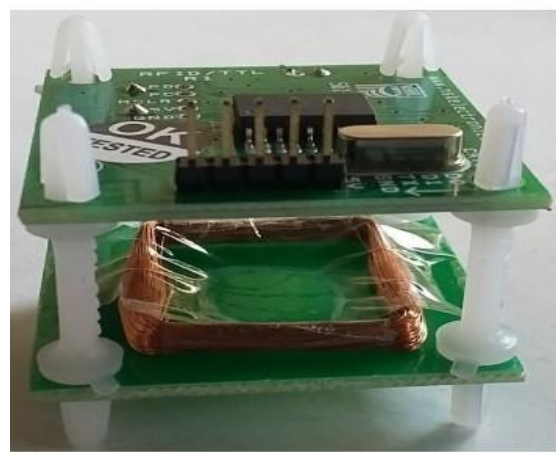

Figure 4: NSK RFID Reader

\section{F. RFID Tag}

RFID tag has 2 types- passive and active tag [4]. Active RFID cards enclose their own power source, benefit of this card is the scanner can be at a very longer distance and still get signal. These devices have restricted life span. Passive RFID cards don't require batteries hence are compact and have limitless life span. The RFID tag and its internal circuitry are as shown in Figure 5. 

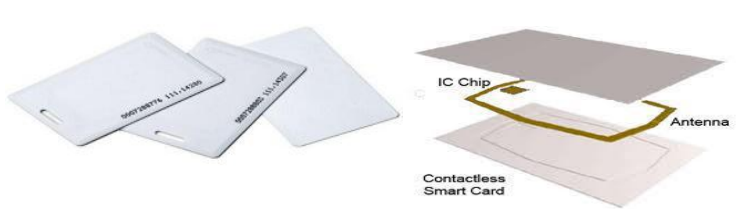

\section{Figure 5: RFID Tag and its Internal Circuitry}

RFID card is a microchip joint which has antenna in a compact package. Figure 7 shows the inner circuitry of RFID tag. The covering is planned to allow RFID card to be attached to track the item [6]. Some extra data like unique serial number is obtained when the tags antenna accept signal from RFID scanner.

Passive cards major advantages and disadvantages are listed below:

\section{Advantages}

1) The card works without a battery.

2) The card is generally less expensive to produce.

3) The card size is much smaller.

Disadvantages

1) With very little distance the card can be read.

2) No need to have sensors which will have electricity for power.

3) The tag remains legible for a very long time, even after the product to which the tag is attached has been sold and is no longer being tracked.

\section{G. Gsm Modem}

Global System for Mobile communication (GSM) is a design used for mobile communication in most of the countries. The SIM800 quad band GSM/GPRS serial modem is shown in Figure 6.

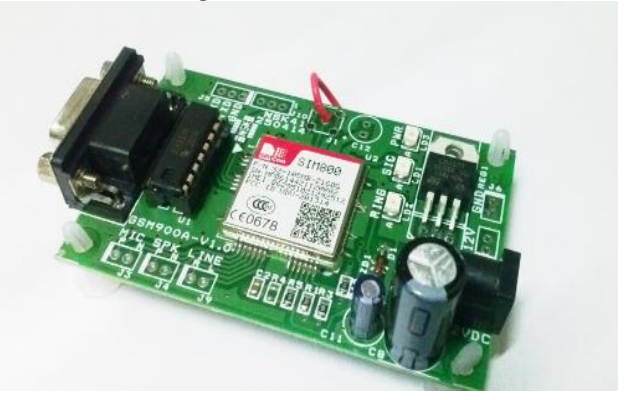

Figure 6: GSM Module 800C

SIM800 is a full quad-band GSM/GPRS system. SIM800H carry quad-band $850 / 900 / 1800 / 1900 \mathrm{MHz}$, it can send voice, SMS and data information with little power consumption. The SIM800 modem has a SIM800 GSM component and RS232 interface which allows simple connection with the computer or laptop using USB to serial connector or to the microcontroller using RS232 to TTL converter. Embedded $\mathrm{C}$ code is written using AT commands.

\section{PROGRAMMING LANGUAGE}

\section{A. Embedded C Programming}

The soul of processor implementation inside each and every embedded system such as mobile phones, TV, digital cameras and washing machine is the Embedded $\mathrm{C}$ programming. Each and every processor will be connected with embedded software. Embedded software decides the functioning of embedded system. Most often used language to program the microcontroller is Embedded $\mathrm{C}$ language. Embedded $\mathrm{C}$ is an addition to $\mathrm{C}$ programming language that provides support for increasing efficient programs for embedded devices. In embedded systems hardware becomes the body, embedded processor act as brain and embedded software forms its soul.

For microcontroller based applications Embedded C is used. Limited resources are used such as RAM, ROM, I/O's on an embedded processor. Embedded $\mathrm{C}$ needs compilers to generate the files to be downloaded to the microcontroller/microprocessor anywhere it wants to run. In this project Embedded $\mathrm{C}$ code is written for ARM7 LPC2148, LCD, RFID, motor and RFID tag to distinguish between the types of vehicle.

\section{FLOW CHART OF THE SYSTEM}

A. Flow chart for automatic vehicle identification for multiple purposes at toll collection system.

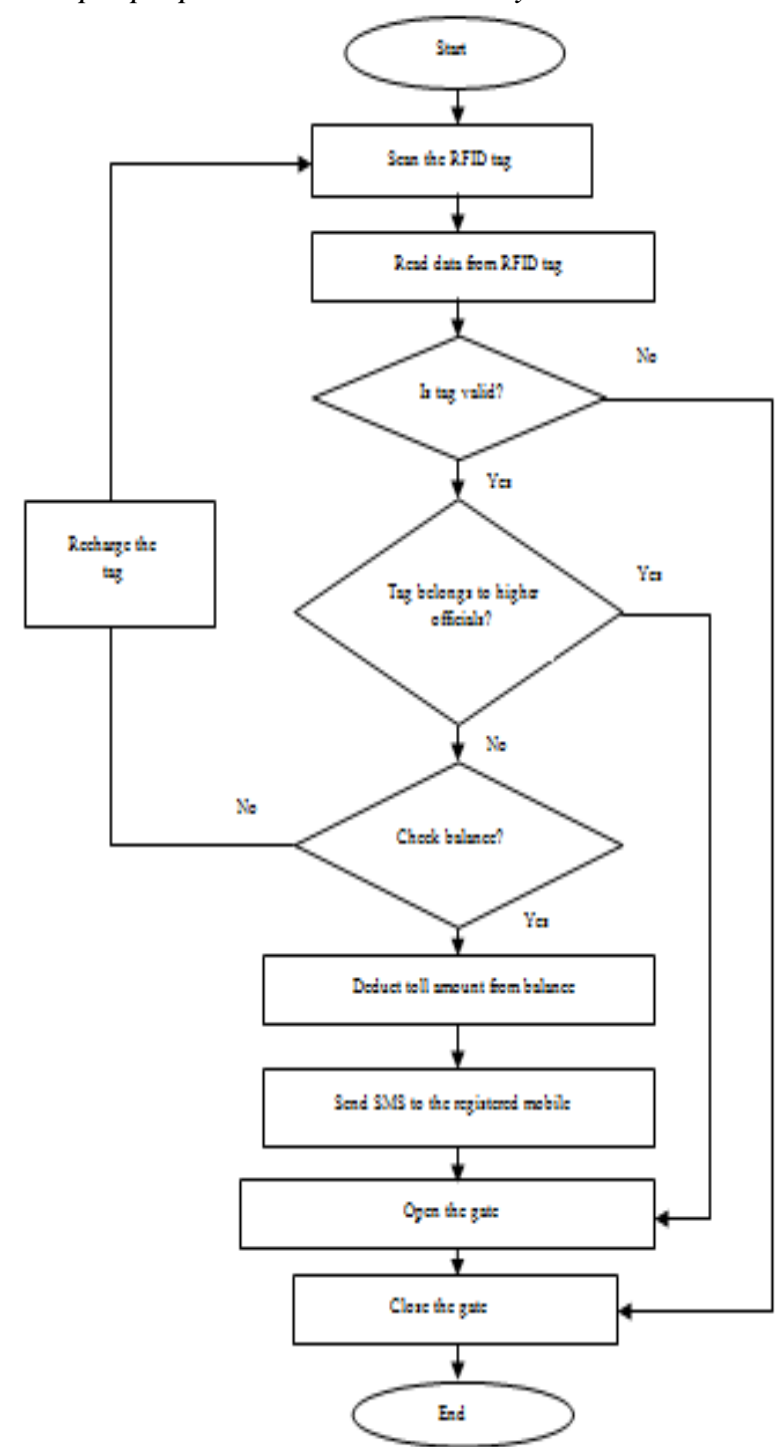

Figure 8: Flow Chart for detection of

Initially RFID tag is scanned and data is read from it. This RFID tag 
number is compared with database to know if it is valid number. If the number is valid then tag number belongs to higher officials or not is determined. If the number belongs to higher officials such as VIP, police, army, minister's vehicle or an ambulance the toll gate is opened directly without deduction of toll amount. If the vehicle does not belong to higher officials and is registered then if the owner has sufficient balance toll amount is deducted and the gate is opened.

Message of amount deducted and balance will be send to owners' mobile number. Flow chart for automatic vehicle identification is shown in Figure 7.

\section{B. Flow Chart for detection of stolen vehicle at toll} collection system

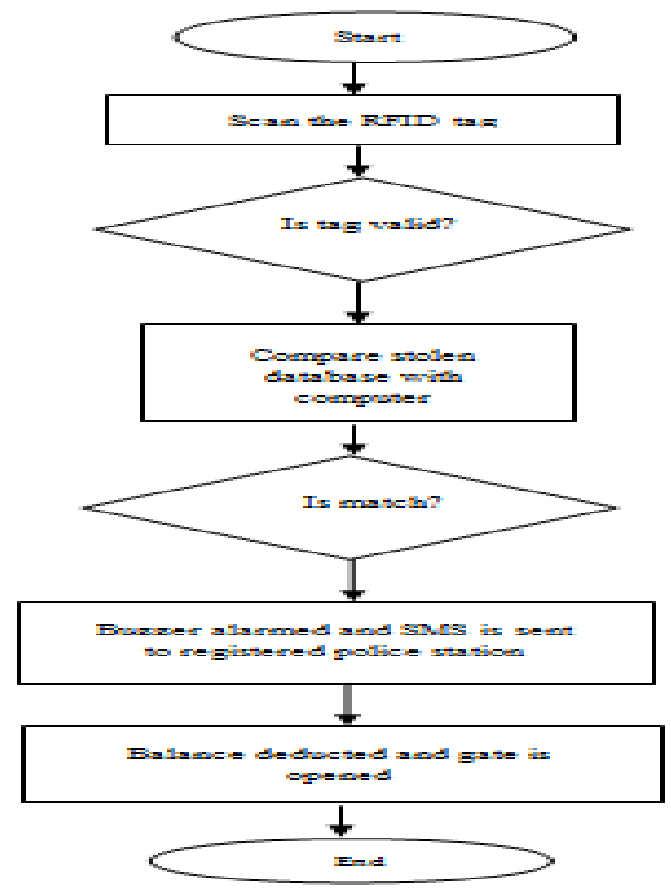

\section{Figure 7: Flow chart for Automatic Vehicle Identification.}

RFID tag is scanned using RFID reader and checked if it is valid or not. If the tag is not valid then the tag number is compared with the stolen database. If it matches then buzzer is alarmed and notification is send to both police station and the owner. The owner gets the message of amount deducted and his balance. Then the toll gate is opened. Figure 8 shows flow chart for detection of stolen vehicle

\section{RESULTS}

Figure 9 depicts the basis hardware implementation of the project which consists of RFID reader, RFID tags, LED's, LCD display, ARM 7 LPC2148, GSM and a buzzer.

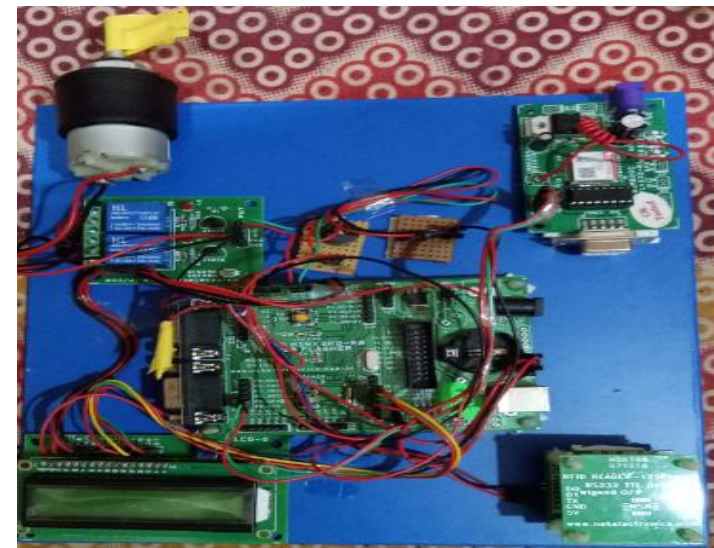

Figure 9: Basic hardware implementation

All RFID tags have unique tag numbers.

Based on tag number present in the database of computer host vehicle is identified as valid (Access general), VIP or stolen. This is displayed on LCD. Message will be send to holder if it is general access vehicle and message will be send to both holder and police station if it is a stolen vehicle which is depicted in Figure 10. The gate will be opened for certain time period and then closed.
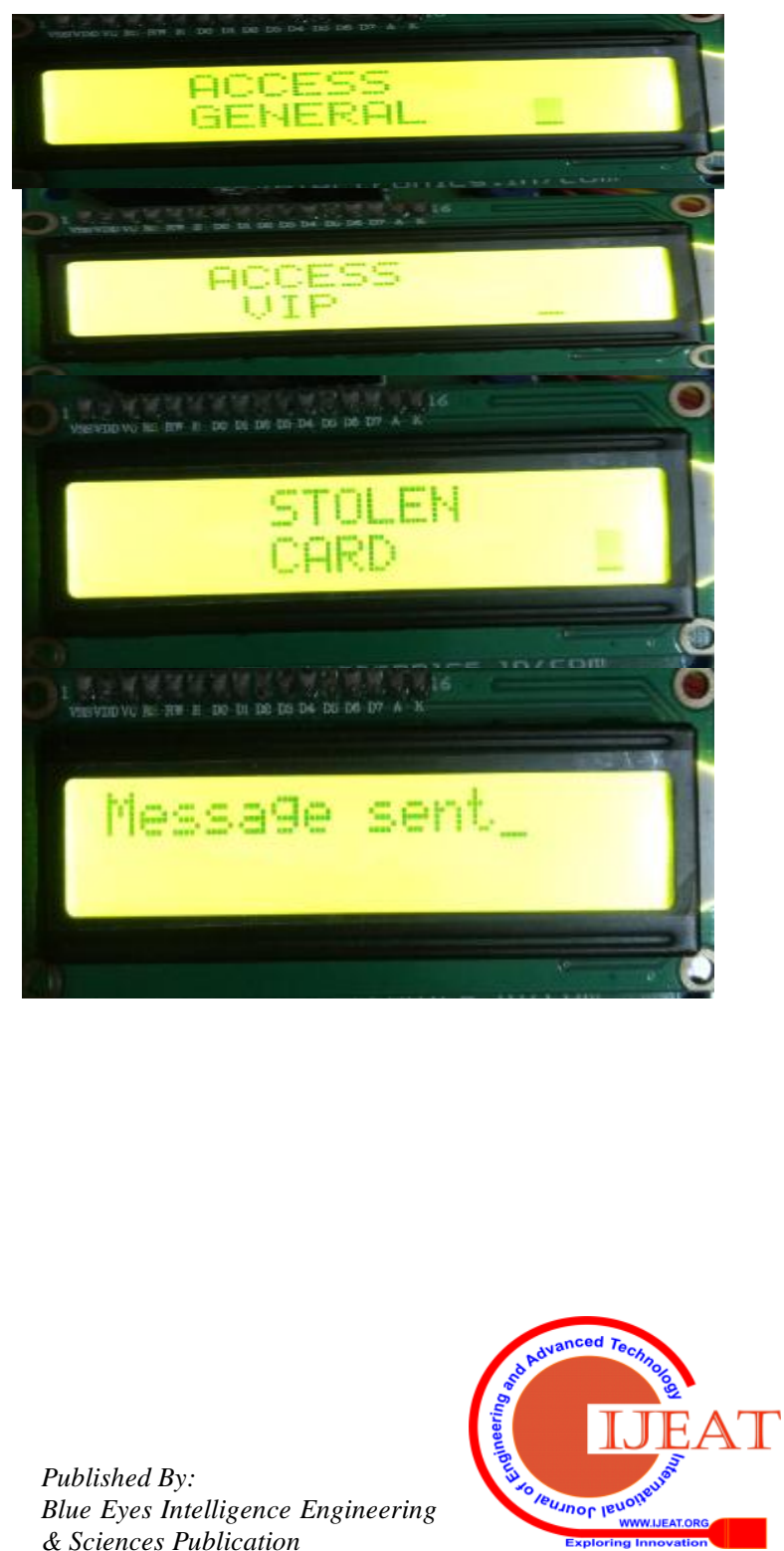

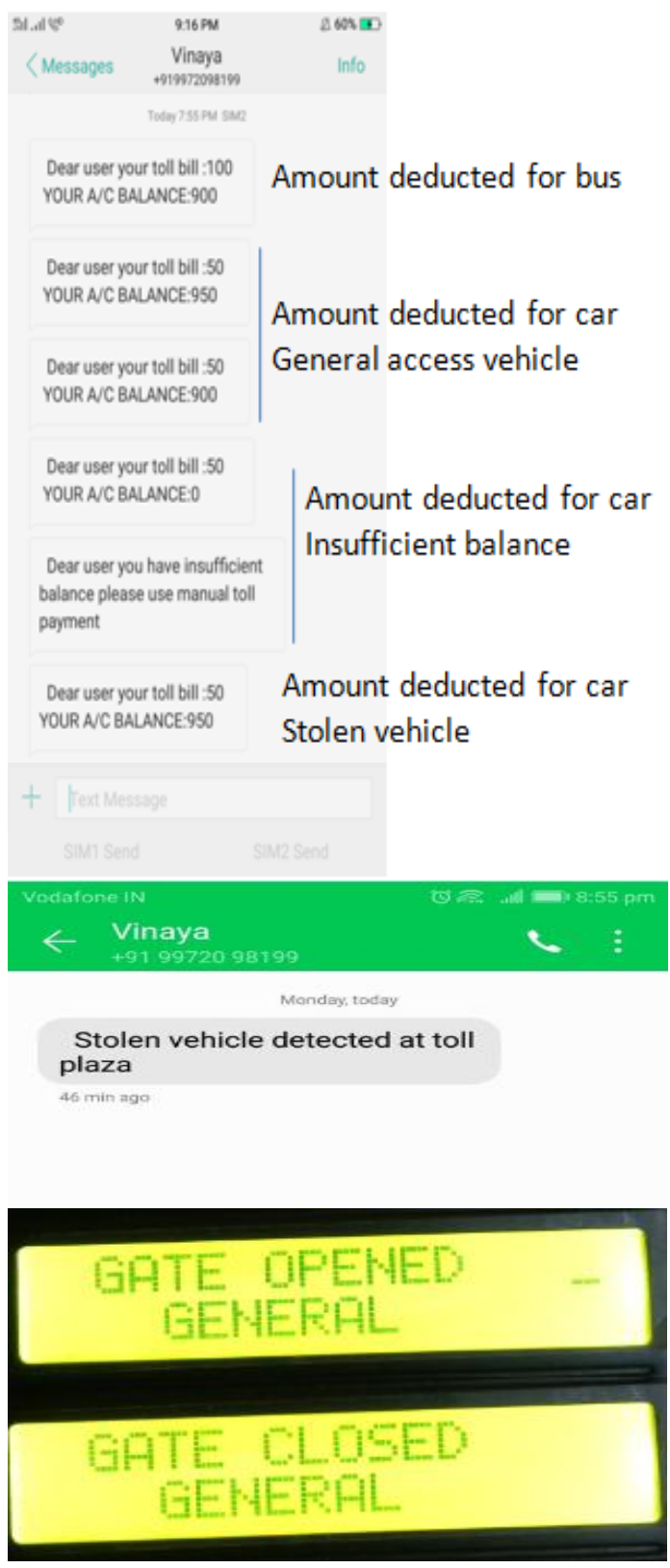

Figure 10: Vehicle type determination using RFID tag and message sent

This paper discusses about the automatic vehicle identification for multiple purposes at toll collection system. RFID technology is used which is highly steady as well as consistent technology. The RFID without human intervention detects the type of vehicle such as registered, higher officials or stolen vehicle. This system eliminates the human interaction at toll booth. Since the system is fully automatic, time consumed at the toll booth is reduced which in turn reduces the fuel consumption, low cost, high secured, reduce noise and toxin emission at toll station, efficient and reduces the traffic. In the proposed system, authorized user who has registered will pay the toll amount, message will be sent to his mobile number and gate is opened. If the vehicle belongs to higher officials then they need not pay the toll amount so, the gate is opened directly. If the vehicle is stolen then the message will be sent to the police station, amount will be deducted from the owners account, buzzer will be alarmed and the gate will be opened. The advantage is that the owner receives the message about the toll amount deducted and his balance. This makes the system more beneficial that the message will be send both to the owner in addition to police station.GSM unit is used to send the message to the mobile so that the owner gets to know how much amount he has paid and there are no chances of paying extra money. The main objective is to keep all vehicles under registration, so that no unregistered vehicle can be used and do immoral works against law.

\section{REFERENCES}

1. Amol A. Chapate, D.D. Nawgaje, "Electronic Toll Collection System Based on ARM", International Journal of Science, Engineering and Technology Research (IJSETR), Volume 4, Issue 1, January 2015.

2. Sangeeta Pandey, Yamuna Rai and Shipra Mishra, "Fully Automated Toll Tax Collection Using RF Technology", VSRD-IJEECE, Vol 2, Issue 6, 2012, 417-420.

3. K. Kamarulazizi, dr.W. Ismail, "Electronic Toll Collection System Using Passive RFID Technology", Journal of Theoretical and Applied Information Technology.

4. Aniruddha Kumawat1, Kshitija Chhandramore, "Automatic Toll Collection system Using Rfid", International Journal of Electrical and Electronics Research, Vol 2, Issue 2, 2014, 64-72.

5. Preeti Giri, Priyanka Jain, "Automated Toll Collection Using RFID Technology", IJARSE, Vol 2, Issue 9, 2013.

6. Zhengang. R and Yingbo. G. (2009). "Design of electronic toll collection system in expressway based on RFID", ESIAT 2009, International Conference on, 2 (4-5July 2009), 779-782.

\section{AUTHORS PROFILE}

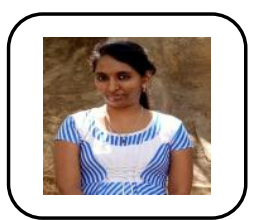

Vinaya Singh $\mathbf{P}$, received her BE (ECE) from VTU, currently she is perusing her MTech (VLSI \& Embedded Systems) from B.N..M. Institute of Technology, VTU. She has worked as an intern in 5square Technologies and she has completed Embedded course.

She has published her paper in International Journal of Advances in Science Engineering and Technology. She has attended 3rd International Conference on Design Innovations for $3 \mathrm{C}$ 's "ComputeCommunicate-Control" (iCdi3C-19) and International Conference on Science, Technology, Engineering and Management (ICSTEM). She participated in tiny chip embedded solutions PSOC workshop and in the technical fest "RENAISSANCE" hosted by IEEE-WIE STUDENT BRANCH. She is currently working in 5square Technologies as a trainer.

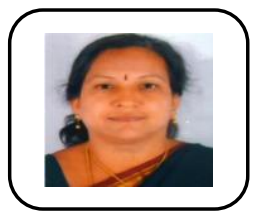

Dr. P. A. Vijaya, Professor \& HOD, Dept. of ECE, BNMIT, Bengaluru. She has done her Ph.D. from IISE, Bangalore. She has 32 years of teaching experience \& 24 years of experience in research. She has published her research papers in leading International Conferences and Journals. She has published her papers in Elsevier Journal, IJCIS International Journal on Computer Engineering and Information Technology, International Journal of Image Processing, International IJRSEEE, IJECCE, IRJET, IJETT, International Journal of Neuroscience and Behavioral Science and many more. She has organized 16 workshops/seminars. She has published a total number of 112 papers.

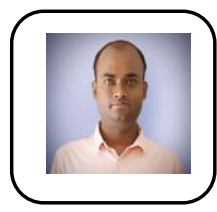

Ravikumar, carries with him of $8+$ years experience in the field of Software development and Training. Having Masters in communication, worked in reputed companies like Honeywell, Delphi. Now working with 5square as Head Trainer..He is a Passionate Programmer and trainer, worked on Embedded Systems, Python, Linux, Device Drivers, System Software and application Development. In past few years actively participating in training on $\mathrm{C}, \mathrm{C}++$, Python, LINUX Kernel, Device Drivers, Machine Learning, etc, for fresher's and working professionals. As a trainer conducted numerous workshops, Tech talks, Training at various symposium, companies and academic institutions across India. 